\title{
A Modified Method for Purifying Gallbladder Epithelial Cells Using Fluorescence-activated Cell Sorting
}

\author{
HAJIME IMAMURA $^{1}$, TOMOHIKO ADACHI ${ }^{1}$, AMANE KITASATO ${ }^{2}$, YUSUKE SAKAI $^{1}$, \\ SHINICHIRO ONO ${ }^{1}$, TAKANOBU HARA ${ }^{1}$, KOJI NATSUDA ${ }^{1}$, AKIHIKO SOYAMA ${ }^{1}$, \\ MASAAKI HIDAKA ${ }^{1}$, MITSUHISA TAKATSUKI ${ }^{1}$, TAMOTSU KUROKI ${ }^{2}$ and SUSUMU EGUCHI ${ }^{1}$ \\ ${ }^{1}$ Department of Surgery, Nagasaki University Graduate School of Biomedical Sciences, Nagasaki, Japan; \\ ${ }^{2}$ Department of Surgery, National Hospital Organization Nagasaki Medical Center, Omura, Japan
}

\begin{abstract}
Background: We have previously reported a procedure for isolating and culturing biliary epithelial cells (BECS). The aim of this study was to reconsider the method for obtaining pure BECs using the mouse gallbladder. Materials and Methods: Cells that were obtained from the gallbladder alone were sorted by fluorescence-activated cell sorting (FACS) for purifying based on the expression of the epithelial cell adhesion molecule (EpCAM). The viability rate was measured based on the negative expression of 7 aminoactinomycin D (7-AAD). Results: More than 75\% of cells from the gallbladder were determined to be pure BECs. An analysis of the EPCAM revealed that $73.3 \%$ of the cells were 7-AAD-negative. Finally, the $0.82 \times 10^{6}$ pure BECs that survived were obtained and seeded on a collagen gel plate. However, these pure BECs showed almost no proliferation. Conclusion: Pure BECs could be accumulated using FACS. However, the number of BECs was insufficient for the culturing process.
\end{abstract}

The performance of basic cell experiments of the liver or biliary tract requires high-purity biliary epithelial cells (BECs). Thus far, BECs have been isolated and cultured from different parts of the biliary tract of humans $(1,2)$ and rodents $(3,4)$. We have previously reported a procedure for isolating and culturing BECs from hamsters; however, the procedure had a disadvantage due to the mechanical and

This article is freely accessible online.

Correspondence to: Susumu Eguchi, MD, Ph.D., Department of Surgery, Nagasaki University Graduate School of Biomedical Sciences, 1-7-1 Sakamoto, Nagasaki 852-8501, Japan. Tel: +81 958197316, Fax: +81 958197319, e-mail: sueguchi@nagasakiu.ac.jp

Key Words: Biliary epithelial cell, isolation, fluorescence-activated cell sorting, mouse gallbladder. enzymatic damage that occurred during the preparation of the biliary tree and potential contamination by non-epithelial cells (e.g. fibroblasts) that could occur during the isolation process (5).

To overcome these problems, we hypothesized that mechanical and enzymatic damage, which was unavoidable during the preparation of the biliary tree, could be prevented if gallbladder cells were used alone. In addition, fluorescence-activated cell sorting (FACS) is considered to be useful for purifying contaminated cell populations. The mouse is the most commonly used animal model for studying human diseases. The genetic characteristics of mice closely resemble those of humans, and many symptoms of human conditions can be replicated. Thus, mice were used instead of hamsters in the present study.

The aim of this study was to reconsider the method for obtaining pure BECs using the mouse gallbladder and to evaluate the effectiveness of FACS in the purification of BECs.

\section{Materials and Methods}

Animals. Male C57BL/6J mice (age: 8-10 weeks) were supplied by the Shizuoka Laboratory Animal Center (Shizuoka, Japan) and were kept under standard laboratory conditions in the Laboratory Animal Center for Biomedical Research, Nagasaki University School of Medicine. The animals had ad libitum access to a standard diet and water. This study was carried out in strict accordance with the recommendations of the Guide for the Care and Use of Laboratory Animals of the National Institutes of Health. The protocol was approved by the Committee on the Ethics of Animal Experiments of Nagasaki University. In the present study, mice were used to obtain gallbladder fragments; the biliary tract - including the intrahepatic and extrahepatic bile ducts - was not used.

The culturing of gallbladder fragments and the isolation of cells. Six mice were used for the collection of gallbladders in a one-time experiment. The gallbladder was resected from the liver and divided into 3-4 fragments. These gallbladder fragments were embedded directly on a collagen gel plate with $3 \mathrm{ml}$ of culture medium. A 

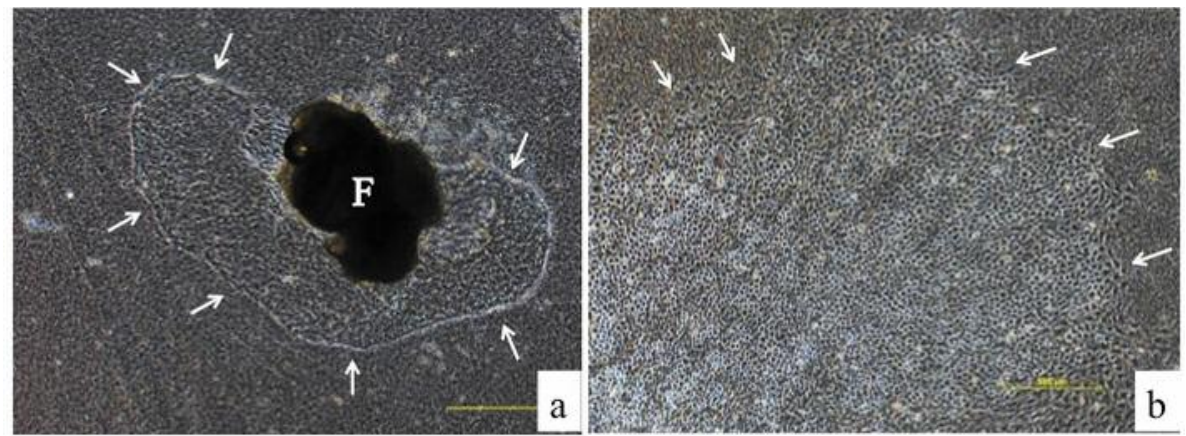

Figure 1. On day 5, the spreading of cells from the fragment $(F)$ was detected on the collagen gel (arrows; magnification: $\times 40)(A)$. The biliary epithelial cells were observed to have spread into a sheet-like formation (arrows) with a cobblestone appearance on day 7 (magnification: $x 40$ ) (B).

collagen gel plate was made using 60 -mm petri dishes with $3 \mathrm{ml}$ of collagen solution for incubation at $37^{\circ} \mathrm{C}$. The collagen solution was made using a $0.3 \%$ acid solution of collagen (Cellmatrix Type I-A, Nittazeratin, Osaka, Japan), Hank's Balanced Salt Solution 10x (HBSS, Sigma-Aldrich, St. Louis, MO, USA) and $0.8 \mathrm{~N} \mathrm{NaOH}$ (8:1:1) under ice-cold conditions. The culture medium was composed of Dulbecco's modified Eagle medium/HamF12 medium (DMEM/Ham F12, Wako, Tokyo, Japan) containing 10\% fetal bovine serum (Invitrogen GIBCO, Carlsbad, CA, USA) and 1\% penicillin-streptomycin (Invitrogen). The culture media were usually changed every 2-3 days. The spreading of cells from the fragment was detected and showed a cobblestone appearance (Figure 1A-B). After 5-7 days of culturing, the fragment was removed with the attached collagen gel under a phase-contrast microscope using forceps (Figure 2). The majority of the BECs that had spread on the gel could be obtained by this process because the epithelial cells expanded in a horizontal direction on the gel, while the fibroblasts expanded into the gel (6). The remnant cells were detached from the collagen gel by digestion for $40-50 \mathrm{~min}$ with $0.05 \%$ collagenase (Wako, Tokyo Japan) in HBSS (Sigma-Aldrich) and were collected by centrifugation at $1000 \mathrm{rpm}$ for $5 \mathrm{~min}$. After washing once with PBS by centrifugation at $1000 \mathrm{rpm}$ for $5 \mathrm{~min}$, the cells were dissociated in $0.05 \%$ trypsin-EDTA (Invitrogen). After incubation for $5 \mathrm{~min}$ at $37^{\circ} \mathrm{C}$, digestion was discontinued by adding culture medium. The cells that were obtained were analyzed by FACS.

Fluorescence-activated cell sorting. The cells were prepared for use with FITC anti-mouse CD326 (epithelial cell adhesion molecule: EpCAM) antibodies (Biolegend, San Diego, CA, USA) and FITC Rat IgG2a.k Isotype Ctrl antibodies (Biolegend). The viability rate of the EpCAM-positive cells was measured based on the negative expression of 7-aminoactinomycin D (7-AAD) Staining Solution (BD Biosciences, Bedford, MA, USA). All samples were pre-incubated for 15 min (the light was shaded during this time). The cells were analyzed and sorted using a FACS Aria II cell sorter (BD Biosciences).

\section{Results}

Isolation and cultivation of EpCAM-positive BECs. A total of $1.5 \times 10^{6}$ cells, which were obtained from the gallbladder alone, were analyzed by FACS. More than $75 \%$ of the cells

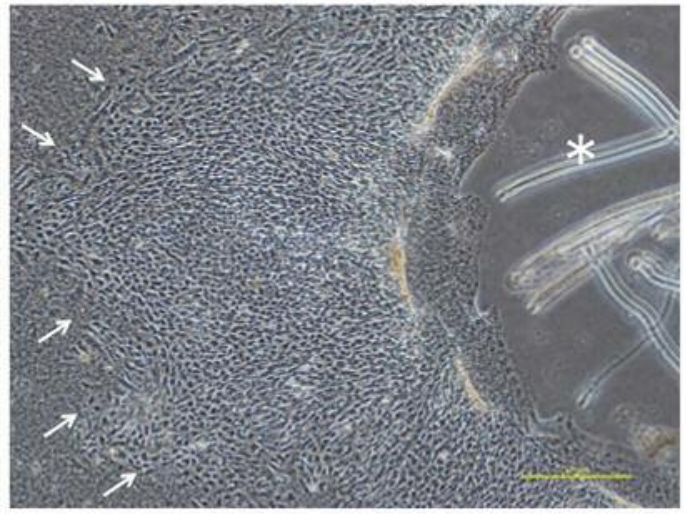

Figure 2. The fragment was removed with an attached collagen gel (*) under a phase-contrast microscope using a forceps after 5-7 days of culturing. The small arrows indicate the peripheral part of the BECs (magnification: $\times 40$ ).

from the gallbladder were pure BECs; they were separated from non-epithelial cells based on their expression of EpCAM (Figure 3). In addition, $73.3 \%$ of the EpCAMpositive cells were 7-AAD-negative (Figure 4). Finally, $0.82 \times 10^{6}$ cells were obtained. These surviving pure BECs were seeded on a collagen gel plate. However, the pure BECs that were separated did not survive for more than 2 days and showed almost no proliferation.

\section{Discussion}

BECs have phenotypes with functional heterogeneity that varies in the different regions of the biliary tree. As a part of the biliary tree, the gallbladder could be a more readilyavailable surgical specimen from which BECs can be obtained in comparison to the intrahepatic or extrahepatic bile duct, even in the clinical setting. Monolayer culturing 

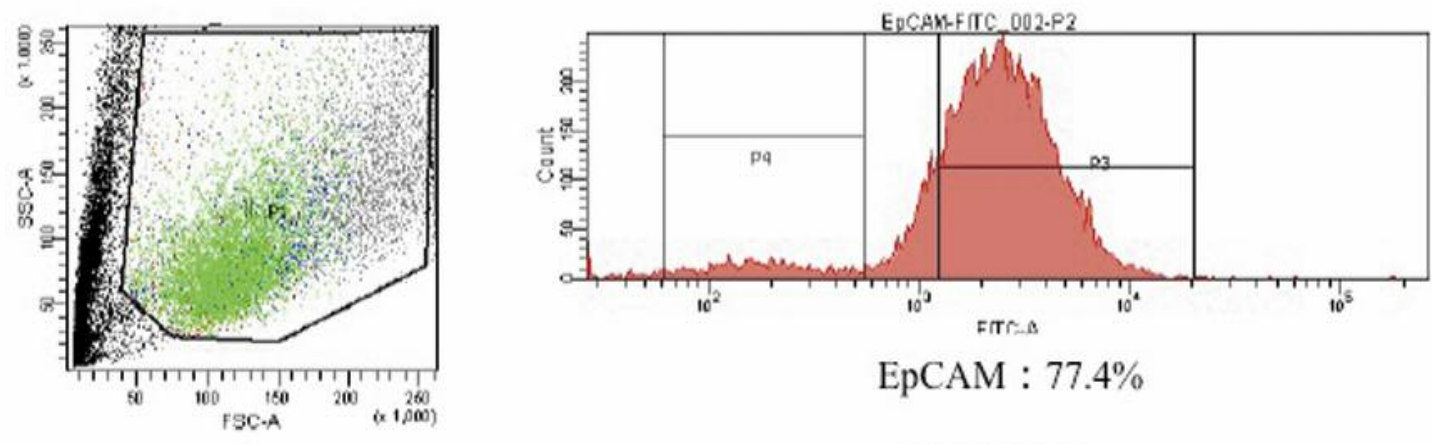

EpCAM : $77.4 \%$

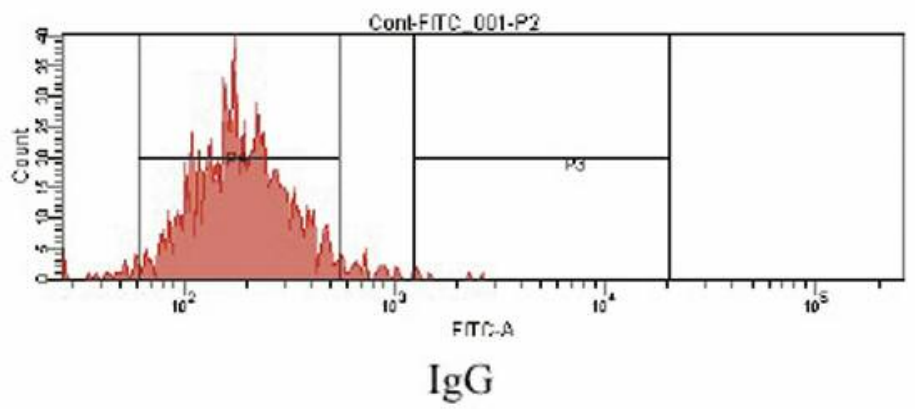

Figure 3. More than 75\% of the cells retained the expression of EPCAM. The EpCAM-positive BECs were successfully separated from non-epithelial cells.

on a collagen-coated culture dish has since long been used for culturing epithelial cells from the gallbladder of humans and experimental animals (6-8). Furthermore, a method for isolating and cultivating pure BECs using the mouse gallbladder has already been reported (3). We previously reported a procedure for isolating and culturing BECs from the biliary tree of hamsters; however, our method was associated with some disadvantages. First, the cultivation of BECs is highly influenced by mechanical and enzymatic damage, which occurs during the preparation of the biliary tree. In addition, contamination by non-epithelial cells can occur during the isolation process (5). We attempted to overcome these disadvantages and obtain high-purity BECs for reliable in vitro culture studies. We next considered using mice instead of hamsters in the present study. The genetic characteristics of mice closely resemble those of humans, and many symptoms of human conditions can be replicated. Furthermore, mice are relatively inexpensive and can be bought in large quantities for research purposes from commercial producers. Lastly, if BECs are to be applied to cancer research or regenerative medicine, it will be necessary to establish a method for isolating high-purity BECs in large quantities. For these reasons, we reconsidered our previous method for obtaining pure BECs using only the gallbladder of mice and attempted to use FACS to purify the contaminated cell population.
In our modified procedure, the process was divided into four steps: preparation, isolation, purification and culture. We only used the gallbladder for preparation which did not cause mechanical damage. This procedure is simple and involves less cellular damage than the previous method that required the biliary tree (5). The isolation process was performed according to our previous method. However, the contamination of non-epithelial cells was assumed to be unavoidable in this situation; we, therefore, attempted to use FACS to purify the contaminated cell population.

The main advantages of FACS-based sorting include the ability to choose between biased and unbiased isolation, the high level of accuracy and the ability to perform highthroughput single-cell isolation (9). The use of FACS for purification in the present study offered some advantages. First, pure BECs could be obtained within a relatively short period in comparison to our previous method. In our previous report, some areas of the BEC sheet, which contained BECs and no contaminating cells, were selectively cut with scissors under a microscope and placed on other collagen gels as a secondary explant for subculture; this also required 2 weeks for primary culturing, with passaging performed every 3 weeks (5). In our modified procedure, it was possible to obtain pure BECs within 7 days. Most of the expanded cells could be used without waste because the isolation process only required the removal of the fragment with the attached collagen gel for FACS. The reason for this 


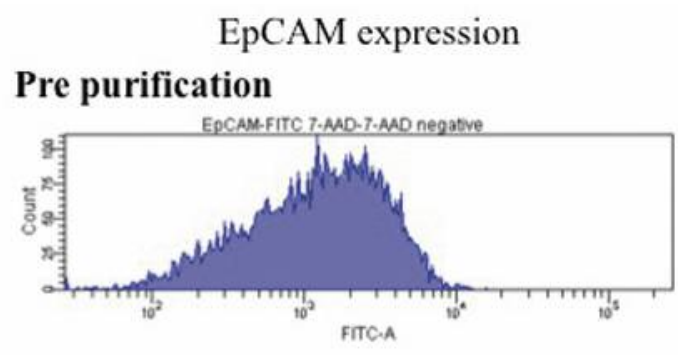

After sorting (EpCAM positive)
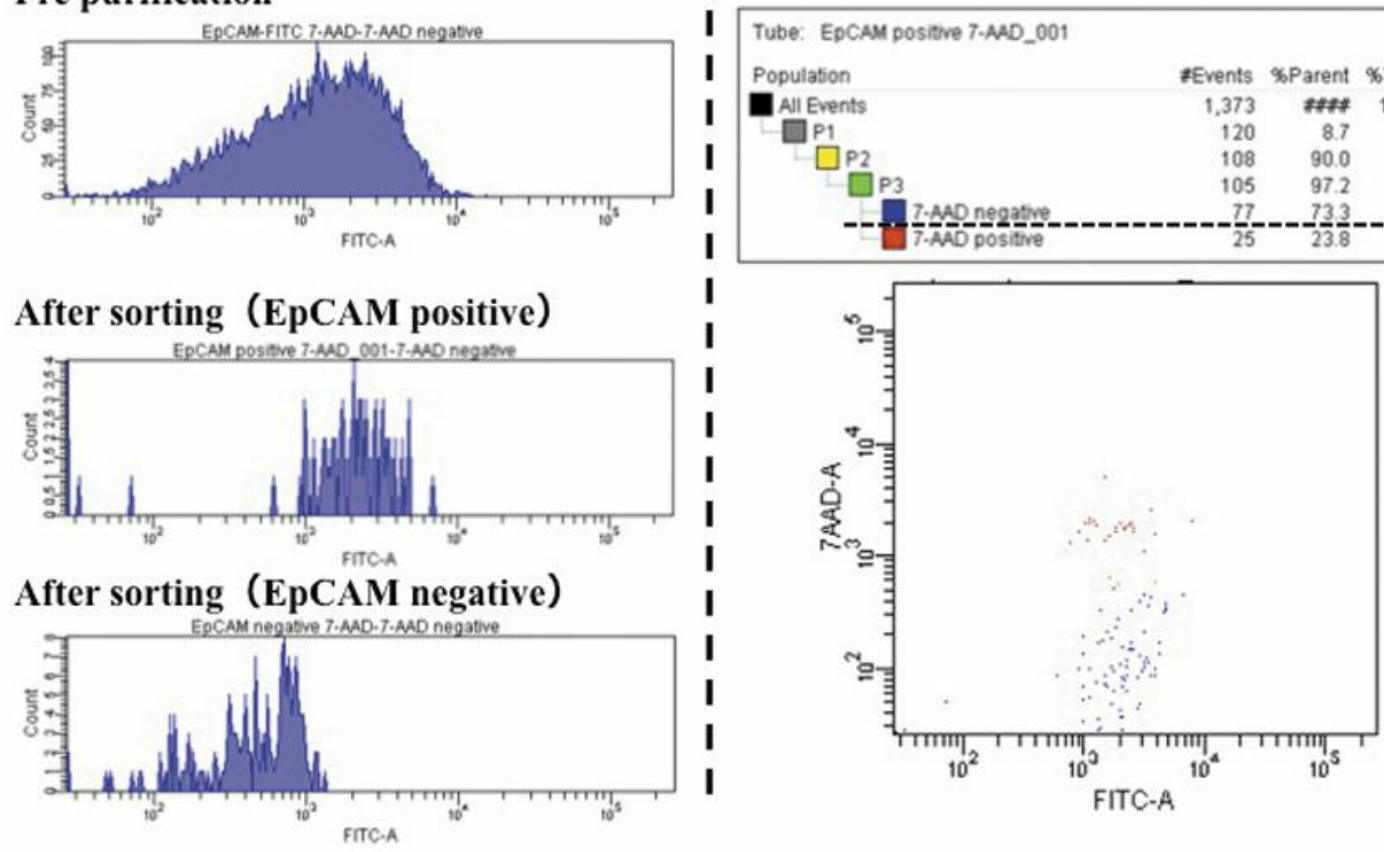

Figure 4. Following the sorting process, 73.3\% of the EpCAM-positive cells were found to be 7-AAD-negative (dotted underline).

was that BECs could be accurately separated from remnant cells, including BECs and other cells, during the sorting process using the specific markers for the cell type of a targeted sample. In this study, EpCAM antibodies were used as a marker of BECs. EpCAM is a cell-surface glycoprotein that is expressed on some normal as well as neoplastic epithelial cells $(10,11)$. In the adult liver, it is reported to be expressed on BECs, but not hepatocytes (12). Furthermore, it has been assumed-based on the sorting results - that some degree of contamination with non-epithelial cells might have occurred if we had not adequately removed the contaminated cell area under phase-contrast microscopy using forceps, as was performed in our previous method.

The BECs that were acquired in the present study showed almost no proliferation; however more than $70 \%$ of the cells were found to be viable based on the results of the 7-AAD analyses. This might be because the number of purified BECs that could be collected from an individual mouse might have been too small to achieve sufficient proliferation. FACS requires a large number of cells in suspension as a starting material, which might affect the yield with respect to cell subpopulations with low abundance (13). To ensure that the cell volume is sufficient for an analysis in a single experiment, we should investigate the duration that is appropriate for primary culturing and the number of gallbladder fragments that should be used in a future study.
In conclusion, pure BECs could be accumulated using FACS. However, the preparation of a significant number of BECs was required for the culturing process. In addition, it might be necessary to improve the method to maintain the cellular function and proliferative ability. Further studies should be performed to obtain BECs that have the ability to proliferate.

\section{Conflicts of Interest}

The Authors declare no conflicts of interest in association with the present study.

\section{References}

1 Auth MK, Keitzer RA, Scholz M, Blaheta RA, Hottenrott EC, Herrmann G, Encke A and Markus BH: Establishment and immunological characterization of cultured human gallbladder epithelial cells. Hepatology 18(3): 546-555, 1993.

2 Strain AJ, Wallace L, Joplin R, Daikuhara Y, Ishii T, Kelly DA and Neuberger JM: Characterization of biliary epithelial cells isolated from needle biopsies of human liver in the presence of hepatocyte growth factor. Am J Pathol 146(2): 537-545, 1995.

3 Katayanagi K, Kono N and Nakanuma Y: Isolation, culture and characterization of biliary epithelial cells from different anatomical levels of the intrahepatic and extrahepatic biliary tree from a mouse. Liver 18(2): 90-98, 1998. 
4 Yang L, Faris RA and Hixson DC: Long-term culture and characteristics of normal rat liver bile duct epithelial cells. Gastroenterology 104(3): 840-852, 1993.

5 Asakawa $\mathrm{T}$, Tomioka $\mathrm{T}$ and Kanematsu $\mathrm{T}$ : A method for culturing and transplanting biliary epithelial cell from syrian golden hamster. Virchows Arch 436(2): 140-146, 2000.

6 Nakanuma Y, Katayanagi K, Kawamura Y and Yoshida K: Monolayer and three-dimensional cell culture and living tissue culture of gallbladder epithelium. Microsc Res Tech 39(1): 7184, 1997.

7 Joplin R, Strain AJ and Neuberger JM: Biliary epithelial cells from the liver of patients with primary biliary cirrhosis: Isolation, characterization, and short-term culture. J Pathol 162(3): 255-260, 1990

8 Parola M, Cheeseman KH, Biocca ME, Dianzani MU and Slater TF: Isolation and characterization of biliary epithelial cells from normal rat liver. J Hepatol 6(2): 175-186, 1988.

9 Dalerba P, Kalisky T, Sahoo D, Rajendran PS, Rothenberg ME, Leyrat AA, Sim S, Okamoto J, Johnston DM, Qian D, Zabala M, Bueno J, Neff NF, Wang J, Shelton AA, Visser B, Hisamori S, Shimono Y, van de Wetering M, Clevers H, Clarke MF and Quake SR: Single-cell dissection of transcriptional heterogeneity in human colon tumors. Nat Biotechnol 29(12): 1120-1127, 2011 .
10 Armstrong A and Eck SL: Epcam: A new therapeutic target for an old cancer antigen. Cancer Biol Ther 2(4): 320-326, 2003.

11 Momburg F, Moldenhauer G, Hammerling GJ and Moller P: Immunohistochemical study of the expression of a Mr 34,000 human epithelium-specific surface glycoprotein in normal and malignant tissues. Cancer Res 47(11): 2883-2891, 1987.

12 de Boer CJ, van Krieken JH, Janssen-van Rhijn CM and Litvinov SV: Expression of ep-cam in normal, regenerating, metaplastic, and neoplastic liver. J Pathol 188(2): 201-206, 1999.

13 Shapiro E, Biezuner T and Linnarsson S: Single-cell sequencingbased technologies will revolutionize whole-organism science. Nat Rev Genet 14(9): 618-630, 2013.
Received December 27, 2016

Revised February 1, 2017

Accepted February 2, 2017 